\title{
Addition of Capecitabine in Breast Cancer First-line Chemotherapy Improves Survival of Breast Cancer Patients
}

\author{
Di Xu1 ${ }^{*}$, Xiu Chen ${ }^{*}$, Xingjiang $\mathrm{Li}^{1}$, Zhixiang Mao², Wenjuan Tang1, Wei Zhang1,3, Li Ding1,3, Jinhai Tang ${ }^{\circledR}$ \\ 1. Department of General Surgery, The First Affiliated Hospital with Nanjing Medical University, Nanjing, 210029, People's Republic of China \\ 2. Department of Oncology, The Affiliated Hospital of Xuzhou Medical University, Xuzhou, 221000, People's Republic of China \\ 3. The Jiangsu Province Research Institute for Clinical Medicine, The First Affiliated Hospital with Nanjing Medical University, Nanjing, 210029, People's Republic of \\ China \\ *These authors have contributed equally to this work.
}

$\triangle$ Corresponding author: Jin-Hai Tang, Department of General Surgery, The First Affiliated Hospital with Nanjing Medical University, 140 Guang-Zhou Street, Nanjing, Jiangsu, China. TEL: (86)025-68307843, E-mail: jhtang@njmu.edu.cn

(C) Ivyspring International Publisher. This is an open access article distributed under the terms of the Creative Commons Attribution (CC BY-NC) license (https://creativecommons.org/licenses/by-nc/4.0/). See http://ivyspring.com/terms for full terms and conditions.

Received: 2018.09.06; Accepted: 2018.11.09; Published: 2019.01.01

\begin{abstract}
Objective: Capecitabine is an antimetabolic fluoropyrimidine deoxynucleoside carbamate drug that can be converted to 5-FU in vivo. Currently, the role of capecitabine in the treatment of advanced breast cancer has been recognized. Also, Several meta-analyses have elucidated the role of capecitabine in the treatment of breast cancer, indicating that taxane-based regimen with capecitabine may be an effective, convenient, and well tolerated regimen in patients with early breast cancer. However, the correlation between capecitabine-based combination first-line chemotherapy and breast cancer survival remains unclear. Here, we present a meta-analysis to systematically evaluate the safety and effectiveness of capecitabine-based combination with first-line chemotherapy treatment in breast cancer.

Methods: We searched Pubmed, Embase, and Medline for relevant studies evaluating pooled estimated hazard ratios of capecitabine in breast cancer patients with the eligible criteria up to June 2018. Fixed and random-effect meta-analyses were conducted based on heterogeneity of included studies.

Results: Overall, 10 articles with 12,872 patients were included in the meta-analysis. Capecitabine-based combination first-line chemotherapy compared with non-combination had significant impacts on disease-free survival $(\mathrm{HR}=0.84,95 \% \mathrm{Cl}: 0.76-0.93 ; \mathrm{P}=0.000)$ and overall survival $(\mathrm{HR}=0.84,95 \% \mathrm{Cl}: 0.74-0.94 ; \mathrm{P}=$ 0.001 ). Also, according to the 3 articles concerning neoadjuvant chemotherapy which included 2534 participants, we found that the addition of capecitabine significantly improved OS (HR $=0.89,95 \% \mathrm{Cl}$ : 0.63-0.86; $P=0.011)$. In the subgroup analysis, TNBC patients got significant benefits with the addition of capecitabine in DFS (HR $=0.77,95 \% \mathrm{Cl}: 0.65-0.92 ; \mathrm{P}=0.004)$ and $\mathrm{OS}(\mathrm{HR}=0.65,95 \% \mathrm{Cl}: 0.51-0.81 ; \mathrm{P}=$ $0.000)$. ER negative patients got significant benefits in OS (HR $=0.73,95 \% \mathrm{Cl}: 0.57-0.93 ; \mathrm{P}=0.012)$. The association of DFS with the addition of capecitabine in Her- patients $(\mathrm{HR}=0.84,95 \% \mathrm{Cl}: 0.71-0.99 ; \mathrm{P}=0.005)$ was significant, as was $\mathrm{OS}(\mathrm{HR}=0.82,95 \% \mathrm{Cl}$ : 0.70-0.95; $\mathrm{P}=0.009)$. Meanwhile, patients receiving capecitabine-based combination first-line chemotherapy underwent less adverse effects especially the grade 3/4 leucopenia than patients with non-combination therapy ( $R R=0.7295 \% \mathrm{Cl}: 0.59-0.86 ; \mathrm{P}=0.000)$.

Conclusion: Capecitabine combined with first-line chemotherapy in the treatment of breast cancer is an effective and safe treatment option and is worthy of clinical application to improve survival of breast cancer patients. In the future, we can continue to carry out relevant researches to explore the upmost appropriate dose of capecitabine for breast cancer.
\end{abstract}

Key words: breast cancer, capecitabine, disease-free survival (DFS), overall survival (OS)

\section{Introduction}

At present, the incidence of breast cancer has ranked first in the malignancy of women. The incidence of breast cancer in Western countries has increased year by year, currently accounting for 30\% 
of all new female tumors [1]. Now, the treatment of breast cancer has entered an era of the comprehensive treatment, and it has formed a mode of treatment that emphasizes both local treatments and systemic treatments of breast cancer. With the development of the systemic adjuvant chemotherapy, the survival outcome of breast cancer patients has been significantly improved.

The international guide with constant updates lately suggests that for breast cancer treatment, anthracycline plus cyclophosphamide, followed by taxane (AC-T), epirubicin plus cyclophosphamide, followed by paclitaxel (EC-P), docetaxel plus doxorubicin and cyclophosphamide (TAC), AC, EC, TC are recommended as the first-line chemotherapy regimen. Besides, among the neoadjuvant chemotherapy regimens, we usually chose programs including both anthracyclines (A) and taxanes (C) according to the latest guide.

Capecitabine, also known as Xeloda, is an antimetabolic fluoropyrimidine deoxynucleoside carbamate drug that can be finally converted to 5-fluorouracil (5-FU) in vivo by thymidine phosphorylase (dThdPase) which can concentrate more in tumour tissues than in normal tissues [2]. Capecitabine has been proven effective as an adjuvant treatment in the metastatic or advanced breast cancer. Namely, it can be used as a rescue treatment $[3,4]$. It can be used alone or in combination with multiple drugs [5,6]. Also, several meta-analyses have elucidated the role of capecitabine in the treatment of breast cancer. One indicated that taxane-based regimen with capecitabine may be an effective, convenient, and well tolerated regimen in patients with early breast cancer [7]. Another meta-analysis indicated that neoadjuvant chemotherapy involving capecitabine can't significantly improve outcomes in breast cancer patients without distant metastasis [8]. However, if the addition of capecitabine in breast cancer first-line chemotherapy can improve the survival of breast cancer patients hasn't been convinced.

Recently, many phase III clinical trials have been undertaken to evaluate the efficacy of capecitabine combined with first-line chemotherapy. In the study of Minckwitz in 2013, it suggested that disease-free survival (DFS) was longer in early non-responders receiving TAC-NX than in those receiving TAC, but it did not significantly improve overall survival (OS) [9]. The trial of Joyce in 2015 found that AC-TX regimen significantly improved the OS rather than DFS [10]. So, we present this meta-analysis to systematically evaluate the safety and effectiveness of capecitabine-based combination first-line chemotherapy in breast cancer patients. We defined the outcomes of DFS and OS as the main endpoints in our meta-analysis. Also, we performed a subgroup analysis such as neoadjuvant and adjuvant chemotherapy results, hormone receptor (ER) and human epidermal growth factor receptor 2 (HER2) status and safety.

\section{Materials and Methods}

\section{Search Strategy}

The following databases were searched for relevant studies: Pubmed (from 1996 to April, 2018), Embase(from 1947 to April, 2016), and Medline, and Cochrane Central Register of Controlled Trials (CENTRAL, from 2000 to April, 2016). We use the following keywords for search: "capecitabine" or "Xeloda" and the expanded MeSH term "Breast Neoplasms" "breast cancer". We restricted the language of literature to English.

\section{Inclusion criteria}

In our meta-analysis, studies should meet the following inclusion criteria to be eligible: (a) studies with breast cancer patients; (b) Patients receive capecitabine-based combination with first-line (neo)adjuvant treatment; (c) being a randomized, open-label, phase III clinical trial; (d) studies that evaluated the efficacy of capecitabine in the neoadjuvant treatment; (e) the reported data of outcomes DFS or OS were sufficient to calculate; (f) reporting hazard risk [HR] with a 95\% confidence interval [CI]. Exclusion criteria included: (a) review, meta-analysis, case report, editorial, conference abstract; (b) the study use single capecitabine in the adjuvant setting.

Two reviewers independently selected the literatures according to the above criteria. In case of disagreement, the third researcher participated in the discussion.

\section{Data extraction}

Data was collected in a standardized form by two independent reviewers. The production of form includes author name; publication year; country; number of patients allocated to each treatment arm; follow-up time; grouping scheme of chemotherapy; clinicopathological characteristics of patients; technique used to measure the efficacy of capecitabine and the evaluation index, etc. The evaluation index of efficacy was disease-free survival (DFS), overall survival (OS). Survival data (hazard ratio [HR], confidence interval $[\mathrm{CI}]$ and $p$ value) were directly taken and used from included studies. In subgroup analysis, we considered ER $>1 \%$ as positive, and her2 $3+$ or FISH+ as positive. 


\section{Data analyses}

Survival data were chosen as evaluation index. Fixed and random-effect meta-analyses were conducted based on heterogeneity of included studies. Data were analyzed using descriptive statistics.

\section{Results}

\section{Characteristics of eligible trials}

Based on the search strategy, we found a total of 1667 potentially relevant articles. After screening the titles and abstracts of these articles, 42 articles were retrieved for detailed evaluation, other 1625 studies were excluded because they were duplicates or irrelevant. After reading the full-text of the 42 articles, 11 articles met our inclusion criteria. However, two articles were published at different times by the same authors concerning different follow-up time, so we selected the article with a longer follow-up of 10.3 years. Finally, 10 articles with 12,872 patients were included in the meta-analysis [9-18]. In the 10 articles, 7 articles were associated with DFS and OS, 1 article was only associated with DFS and 2 articles was only associated with OS. Flowchart of literature selection was presented in Figure 1. All the included articles adopted first-line chemotherapy. Of the 12872 participants, 7,054 received capecitabinebased combination (neo)adjuvant chemotherapy regimens, and 7,018 received capecitabine-free combination (neo)adjuvant chemotherapy regimens. The range of median follow-up was 5 to 6.2 years. Characteristics of studies are shown in Table 1.

\section{Meta-analysis of the primary endpoint}

Disease-free survival was the primary endpoint. Fixed-effect meta-analyses were conducted based on heterogeneity of included studies ( $\mathrm{p}=0.058, \mathrm{I}^{2}=46.8 \%$ ). The HRs for DFS were reported in eight RCTs that included 11,081 participants. We found that DFS was significantly improved in the capecitabine arm versus the non-capecitabine arm (HR $=0.84,95 \% \mathrm{CI}$ : $0.76-0.93 ; \mathrm{P} \approx 0.000$ ) (Figure 2). No publication bias was found.

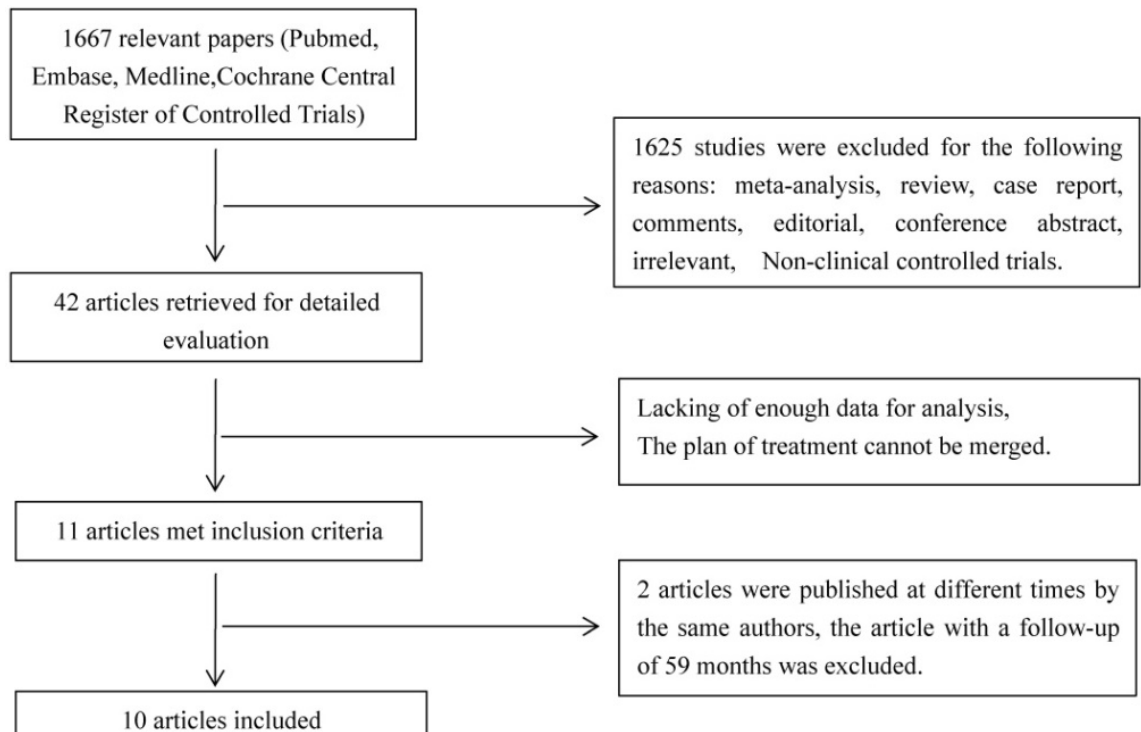

Figure 1. Flow chart of literature selection

Table 1. Characteristics of studies

\begin{tabular}{|c|c|c|c|c|c|c|c|c|}
\hline & Author & Year & Country & Number of patients & Follow-up year & Primary endpoint & Neo/cho & Arms of the study \\
\hline 1 & Minckwitz & 2014 & Germany & 1408 & $5 y$ & DFS,OS & $\mathrm{Neo}$ & EC-T,EC-TX,EC-T-X \\
\hline 2 & Joyce & 2015 & American & 2611 & $6.2 y$ & DFS,OS & & AC-T,AC-XT \\
\hline 3 & Mo"bus & 2017 & Germany & 2994 & $5 y$ & DFS,OS & & EPC,EC-PwX \\
\hline 4 & Minckwitz & 2013 & Germany & 622 & $62 \mathrm{~m}$ & DFS,OS & $\mathrm{Neo}$ & TAC-NX,TAC \\
\hline 5 & Joensuu & 2011 & Finland & 1500 & $59 \mathrm{~m}$ & DFS & & T-CEF,TX-CEX \\
\hline 6 & N. Masuda & 2017 & Japan & 887 & $5 y$ & DFS,OS & & ATX,AT \\
\hline 7 & Joensuu & 2017 & Finland & 1500 & $10.3 y$ & Os & & T-CEF,TX-CEX \\
\hline \multirow[t]{2}{*}{8} & Furlanetto & 2016 & Germany & 173 & $5 y$ & DFS,OS & & ECT,ECT-X \\
\hline & Furlanetto & 2016 & Germany & 382 & $5 y$ & DFS,OS & & ECT,ECT-X \\
\hline 9 & Ohno & 2013 & Japan & 504 & $5 y$ & DFS,OS & $\mathrm{Neo}$ & neo-FEC+T,FEC+T-X \\
\hline 10 & Hatschek & 2011 & Sweden & 291 & $3.5 y$ & OS & & ET,TEX \\
\hline
\end{tabular}

DFS: Disease-free survival; OS: Overall survival; Neo: Neoadjuvant chemotherapy; cho; E: Epirubicin; A: Adriamycin; C: Cyclophosphamide; T: Taxol; P: Paclitaxel; X: Capecitabine 

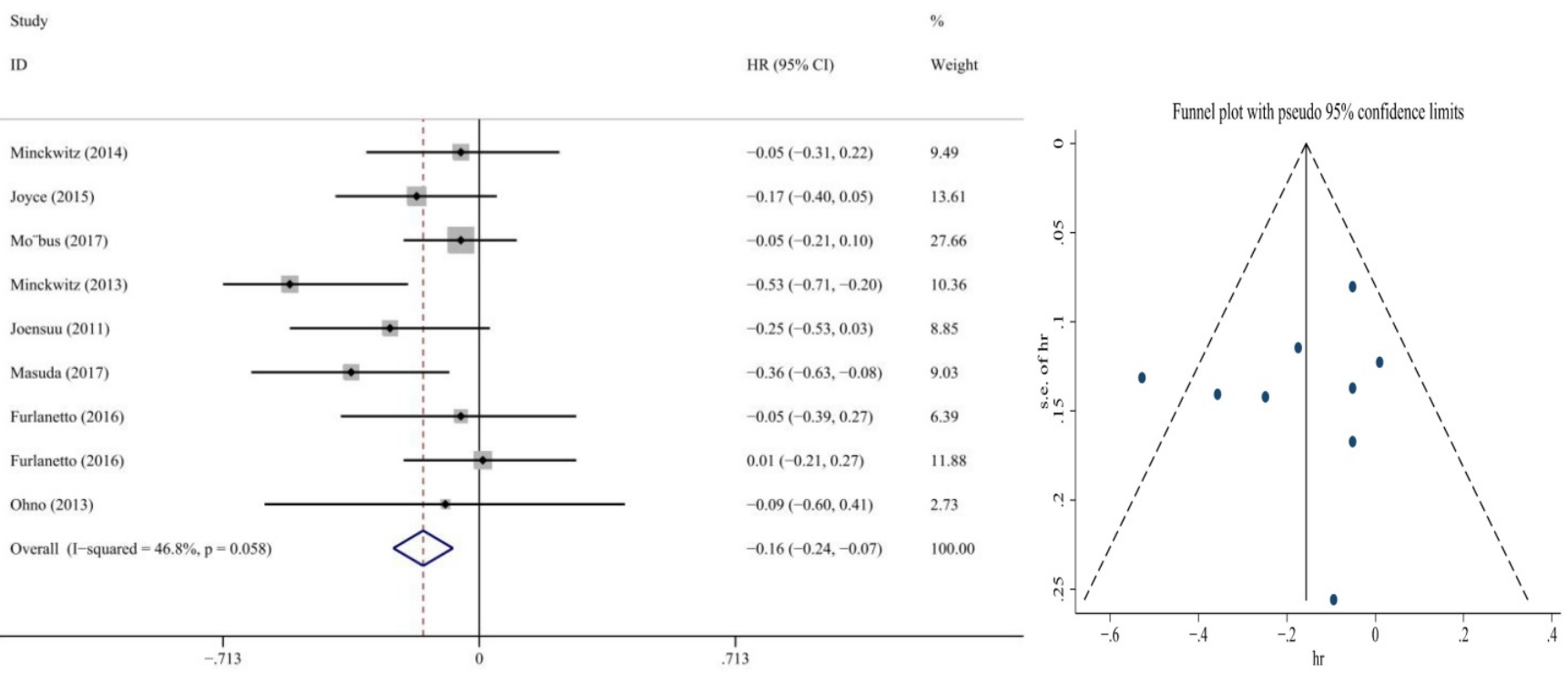

Figure 2. Forest plot and funnel chart of meta-analysis on the disease-free survival for the addition of capecitabine to first-line chemotherapy
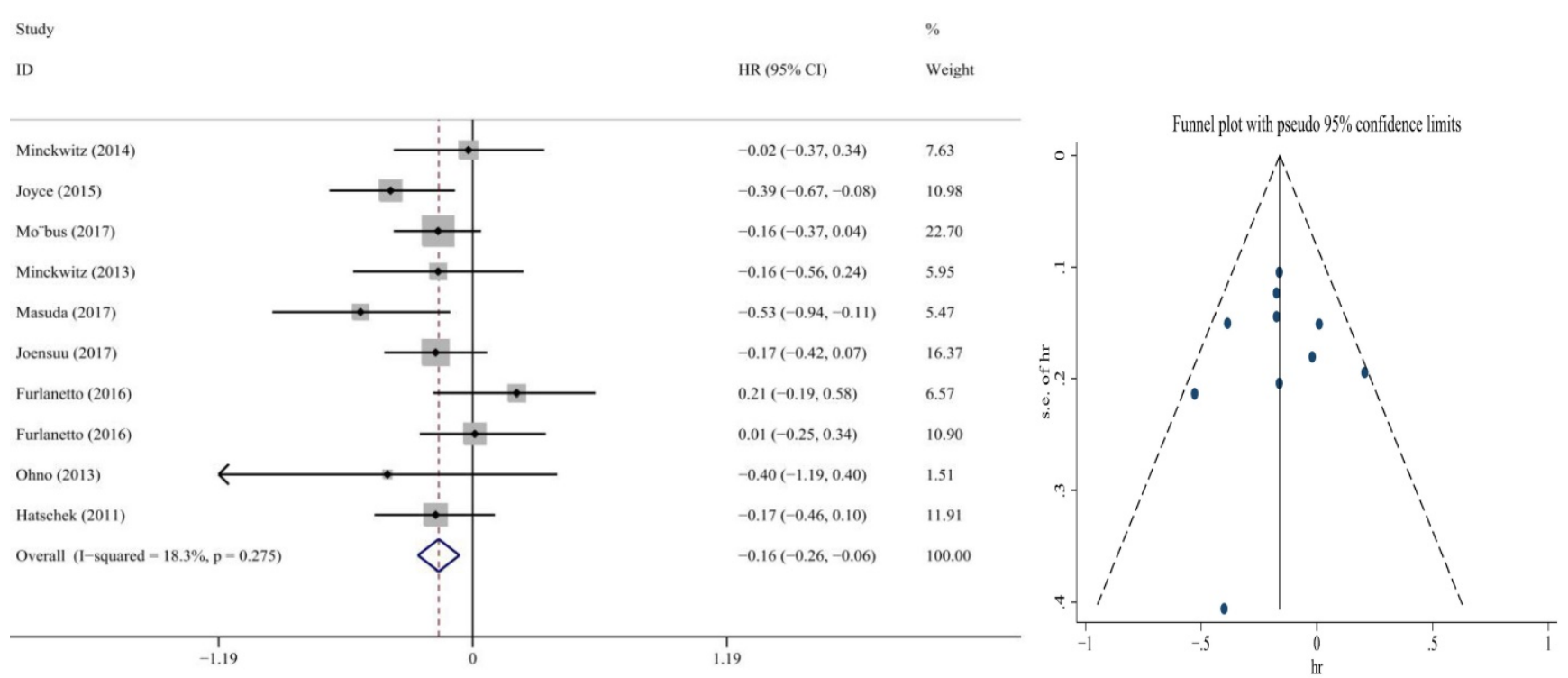

Figure 3. Forest plot and funnel chart of meta-analysis on the overall survival for the addition of capecitabine to first-line chemotherapy

\section{Meta-analysis of the second endpoint}

Overall survival was the second endpoint. Fixed-effect meta-analyses were conducted based on heterogeneity of included studies ( $\left.\mathrm{p}=0.275, \mathrm{I}^{2}=18.3 \%\right)$. The HRs for OS were reported in nine RCTs that included 11,372 participants. In terms of the comparison between the capecitabine arm and the non-capecitabine arm, there was significant differences in OS (HR $=0.84,95 \%$ CI: 0.74-0.94; $\mathrm{P}=$ 0.001) (Figure 3). No publication bias was found.

\section{Subgroup analysis}

In the subgroup analysis, we divided them into capecitabine combined with adjuvant chemotherapy and capecitabine combined with neoadjuvant chemotherapy.
In the 3 articles of neoadjuvant chemotherapy which included 2534 participants, we found that the benefit in capecitabine group was the same as non-capecitabine group in DFS $(\mathrm{HR}=0.76,95 \% \mathrm{CI}$ : $0.41-0.90 ; \mathrm{P}=0.167)$ by random effect $\operatorname{model}(\mathrm{p}=0.032$, $\left.\mathrm{I}^{2}=70.8 \%\right)$, but it significantly improved OS (HR = 0.89, 95\% CI: $0.63-0.86 ; \mathrm{P}=0.011)$ by fixed effect model $\left(p=0.662, I^{2}=0.00 \%\right)$ (Figure 4,5$)$. No publication bias was found.

In addition, the rest articles were concerning about capecitabine combined with adjuvant chemotherapy. The fixed-effect model demonstrated that capecitabine elicited great benefits in DFS $(\mathrm{HR}=0.88$, 95\% CI: 0.78-0.97; $\mathrm{P}=0.011)$ and $\mathrm{OS}(\mathrm{HR}=0.83,95 \%$ CI: 0.73-0.94; $\mathrm{P}=0.002$ ) (Figure 6, 7). No publication bias was found. 

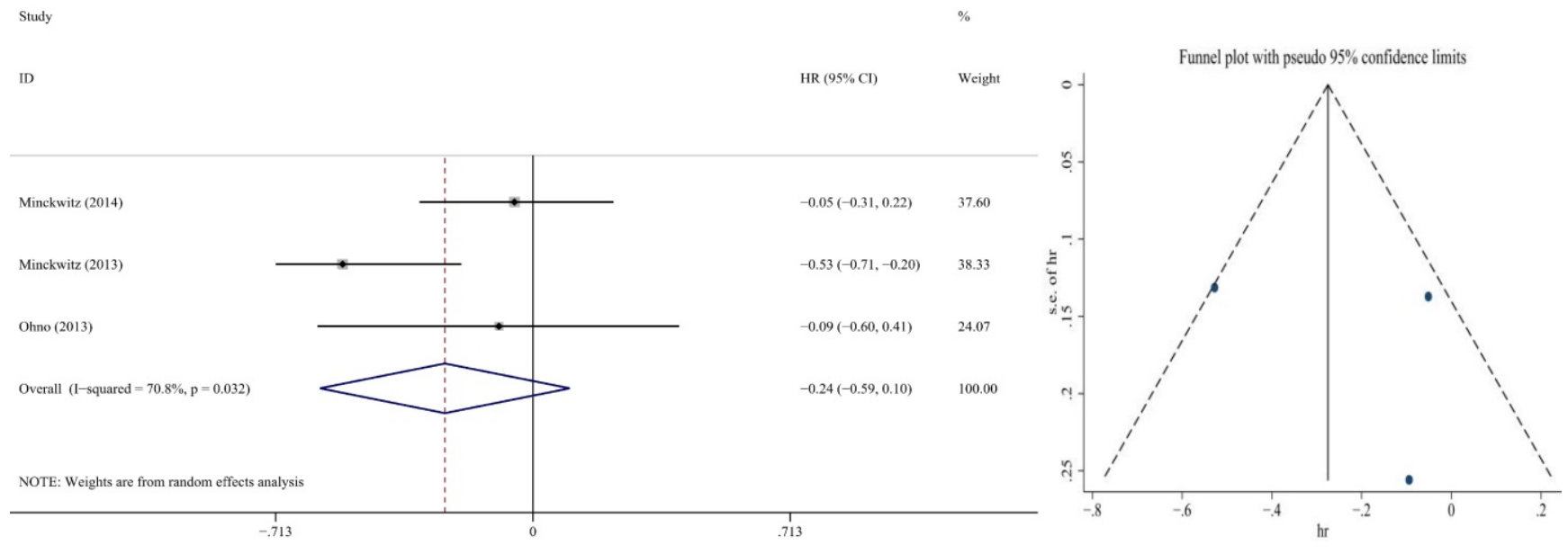

Figure 4. Forest plot and funnel chart of meta-analysis on the disease-free survival for neoadjuvant chemotherapy

Study

ID

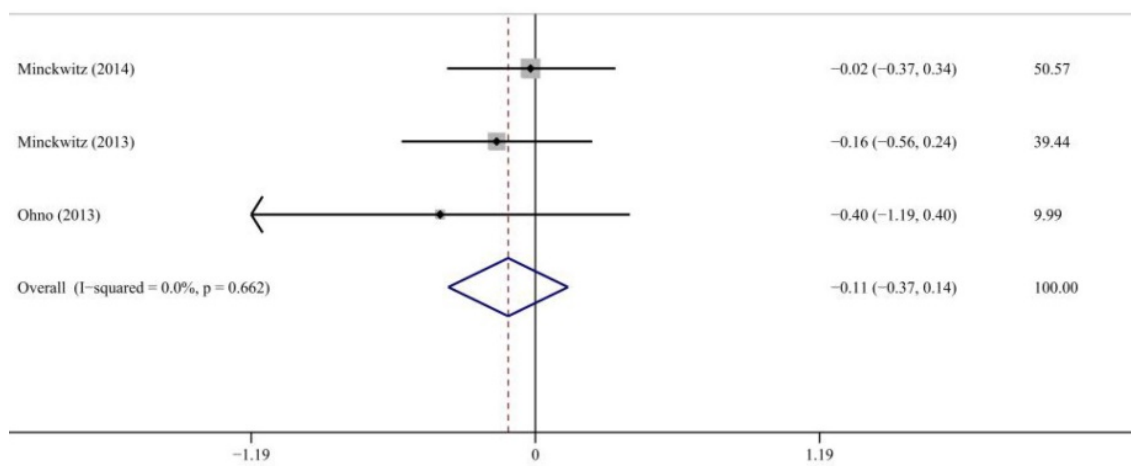

HR $(95 \%$ Cl) Weight

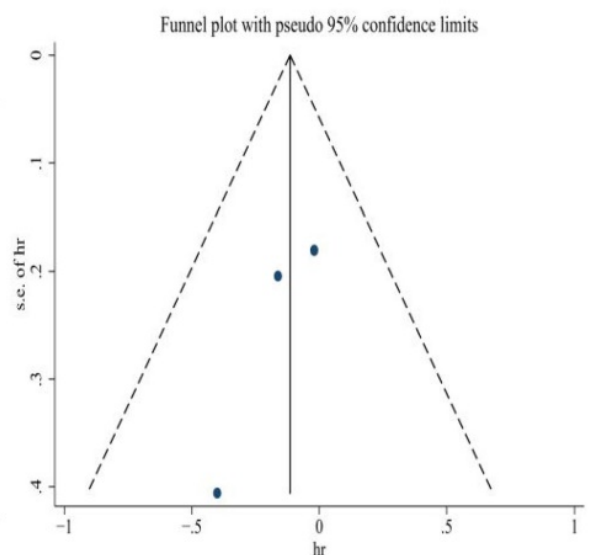

Figure 5. Forest plot and funnel chart of meta-analysis on the overall survival for neoadjuvant chemotherapy

Study

ID

Joyce (2015)

Mo'bus (2017)

Joensuu (2011)

Masuda (2017)

Furlanetto (2016)

Furlanetto (2016)

Overall $(I-$ squared $=15.1 \%, p=0.317)$

$-635$
HR $(95 \% \mathrm{CI}) \quad$ Weight

$-0.17(-0.40,0.05)$

$-0.05(-0.21,0.10)$

$-0.25(-0.53,0.03)$

$-0.36(-0.63,-0.08)$

$-0.05(-0.39,0.27)$

$0.01(-0.21,0.27)$

$-0.12(-0.22,-0.03)$

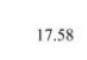

17.58

35.73
11.43

11.66

8.25

15.35

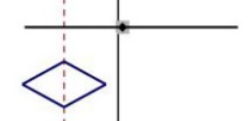

100.00

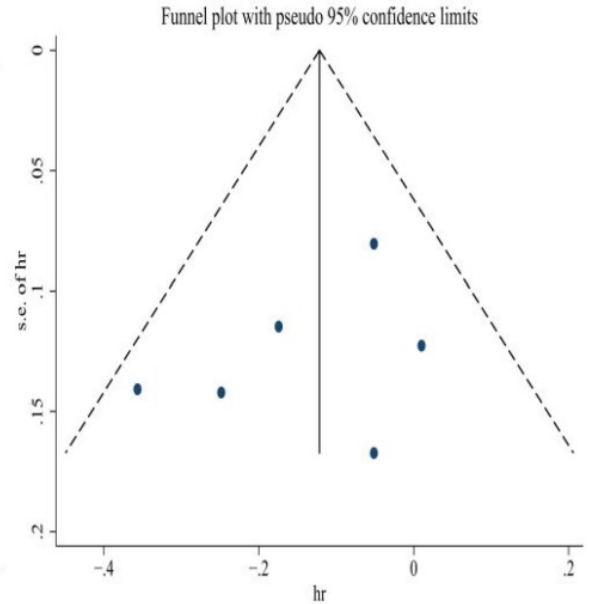

Figure 6. Forest plot and funnel chart of meta-analysis on the DFS for adjuvant chemotherapy 

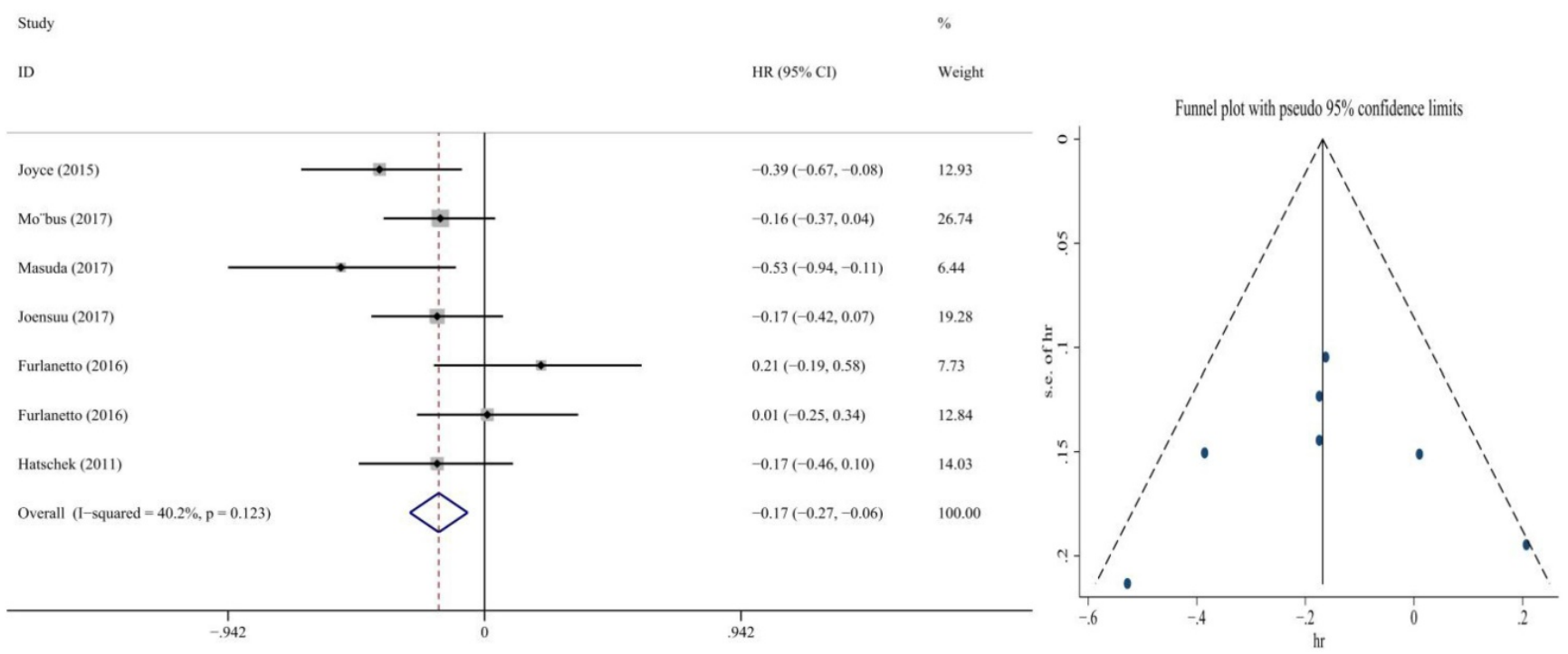

Figure 7. Forest plot and funnel chart of meta-analysis on the OS for adjuvant chemotherapy

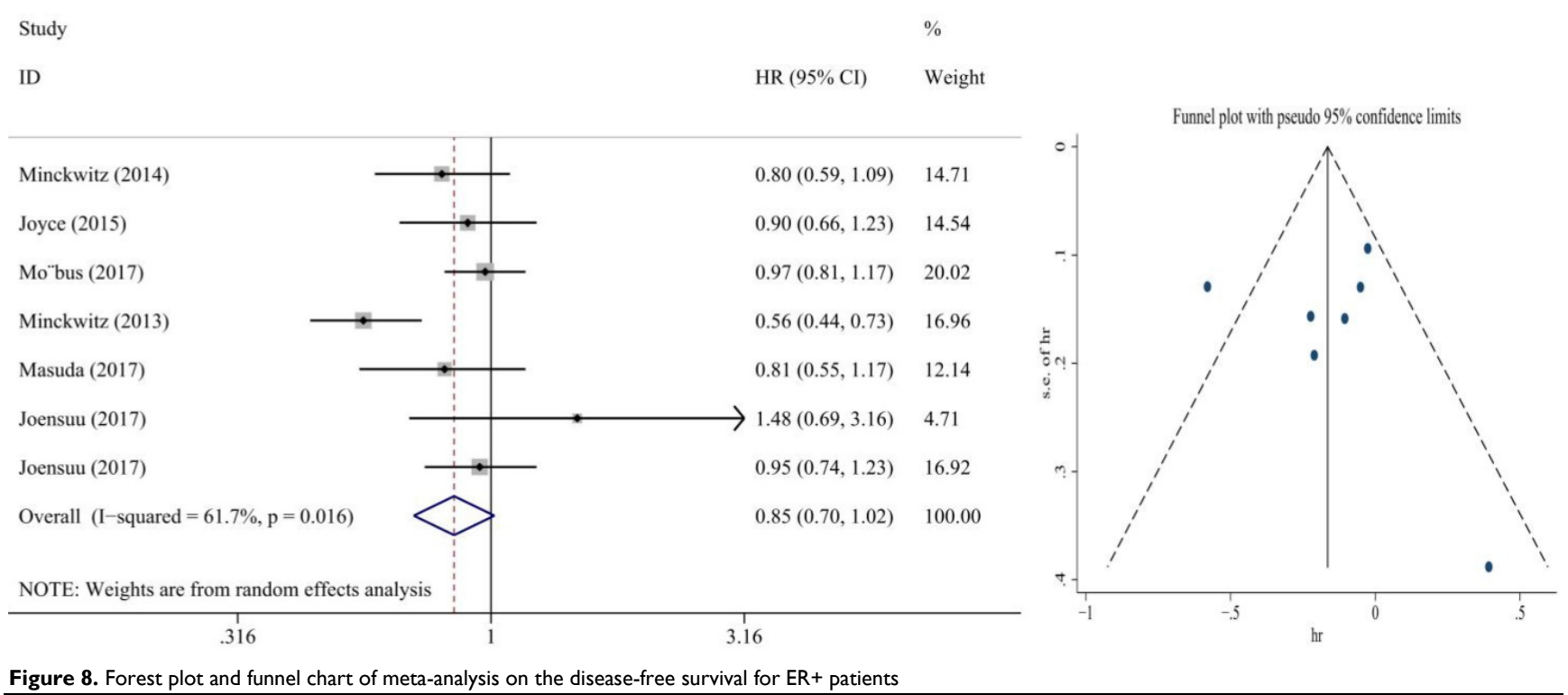

Also, we performed subgroup analysis of DFS and OS according to ER and HER2 status. We defined ER $>1 \%$ was positive, her2 $3+$ or FISH + was positive and we found significant difference in this subgroup. We extracted the included literature data and found that 6 articles analyzed subgroup data. For the ER positive status, no benefit was observed with the addition of capecitabine in DFS (HR $=0.85,95 \% \mathrm{CI}$ : $0.70-1.02 ; \mathrm{P}=0.073)$ and $\mathrm{OS}(\mathrm{HR}=0.86,95 \% \mathrm{CI}$ : 0.73-1.02; $\mathrm{P}=0.079$ ) (Figure 8, 9). In ER negative patients, the addition of capecitabine also didn't significantly improve DFS (HR $=0.89,95 \%$ CI: 0.77-1.02; $\mathrm{P}=0.098)$, however, significant benefit was observed in OS (HR $=0.73,95 \%$ CI: $0.57-0.93 ; \mathrm{P}=$ 0.012) (Figure 10, 11). In the subgroup according to Her2 status, the the addition of capecitabine had no significant benefit in Her2+ patients for DFS (HR =
0.86, 95\% CI: 0.71-1.03; P $=0.096)$ and $\mathrm{OS}(\mathrm{HR}=0.86$, 95\% CI: 0.62-1.19; $\mathrm{P}=0.359$ ) (Figure 12, 13). No publication bias was found. However, In Herpatients, the association with DFS (HR $=0.84,95 \% \mathrm{CI}$ : 0.71-0.99; $\mathrm{P}=0.005)$ was significant, as was OS $(\mathrm{HR}=$ 0.82, 95\% CI: 0.70-0.95; P = 0.009) (Figure 14, 15). In triple negative patients, the analysis revealed that capecitabine improved the DFS (HR $=0.77,95 \% \mathrm{CI}$ : $0.65-0.92 ; \mathrm{P}=0.004)$ and $\mathrm{OS}(\mathrm{HR}=0.65,95 \% \mathrm{CI}$ : $0.51-0.81 ; \mathrm{P}=0.000$ ) (Figure 16, 17). No publication bias was found.

\section{Safety analysis}

In all included articles, 4 articles compared safety differences between the capecitabine group and the non-capecitabine group. So we analyzed 8 common hematological and non-hematological toxicities, 
including febrile neutropenia grade3/4, neutropenia grade $3 / 4$, leucopenia grade $3 / 4$, thrombocytopenia, anemia grade $3 / 4$, diarrhea, vomiting and hand-foot syndrome.
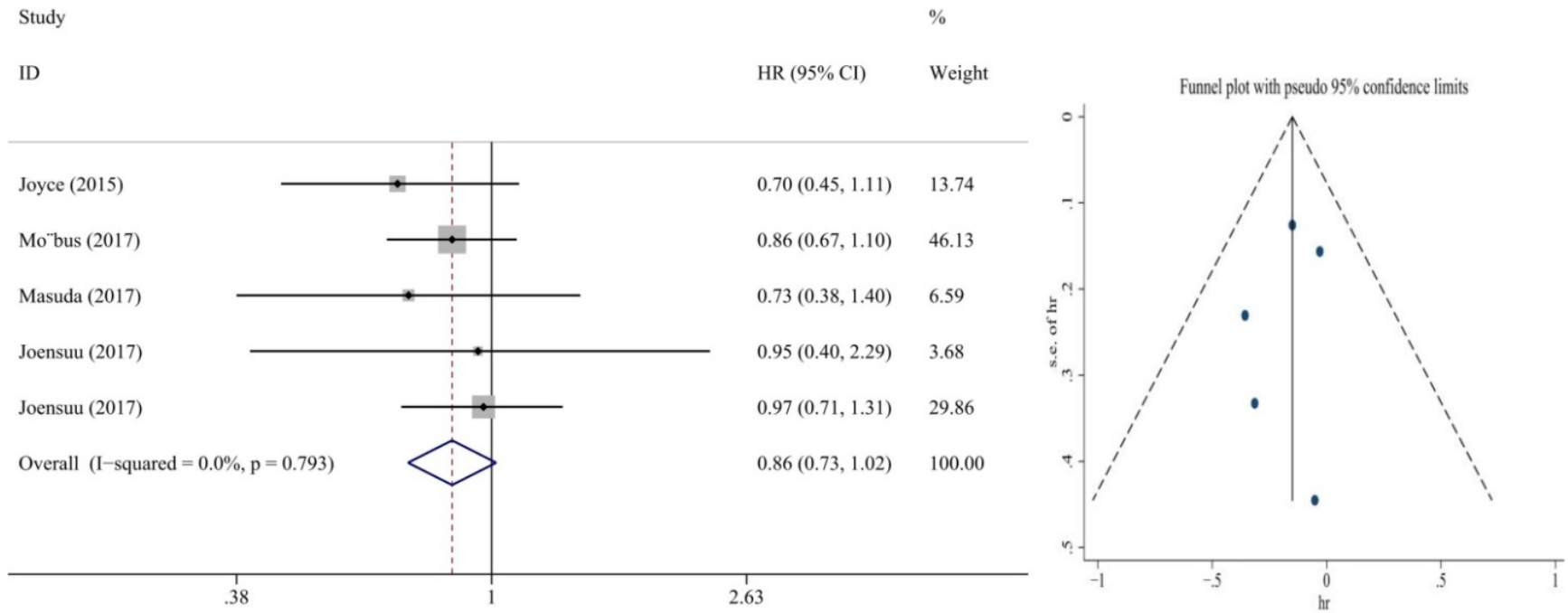

Figure 9. Forest plot and funnel chart of meta-analysis on the overall survival for ER+ patients

Study

ID

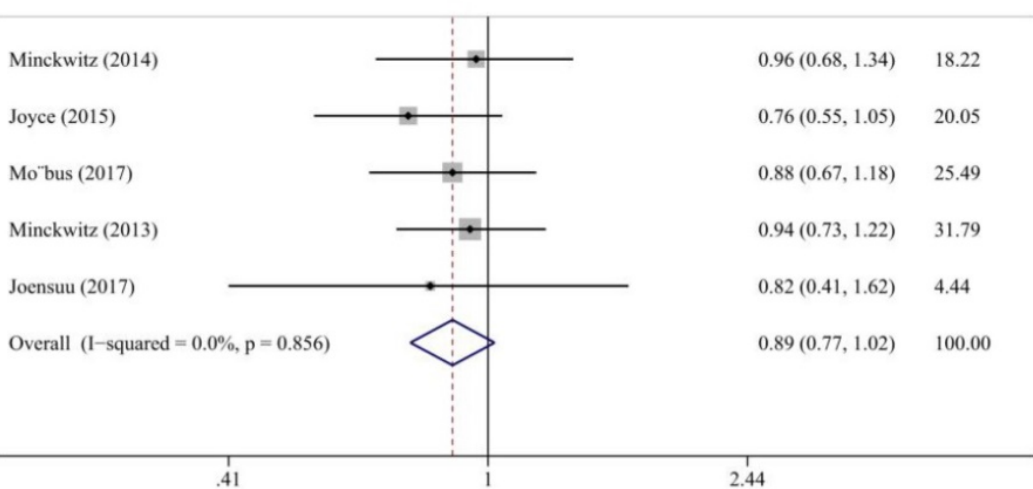

$\%$

HR $(95 \% \mathrm{CI}) \quad$ Weight

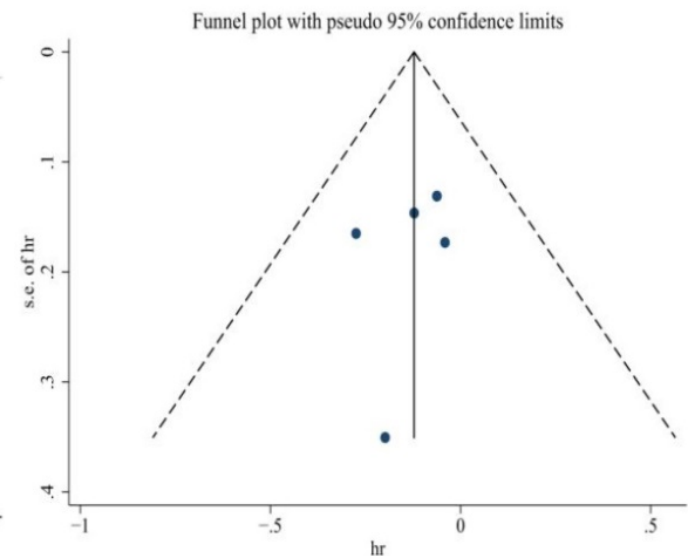

Figure 10. Forest plot and funnel chart of meta-analysis on the disease-free survival for ER- patients

Study

ID

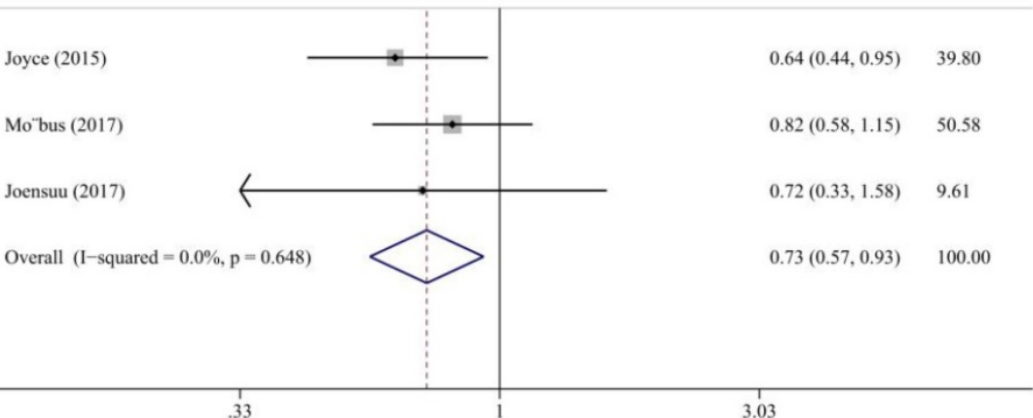

$\%$

HR $(95 \% \mathrm{CI}) \quad$ Weight $\quad$ Funnel plot with pseudo $95 \%$ confidence limits

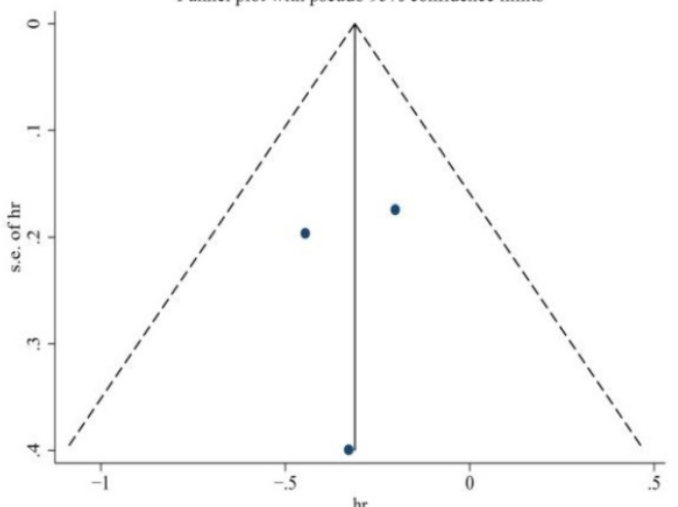

Figure 11. Forest plot and funnel chart of meta-analysis on the overall survival for ER- patients 

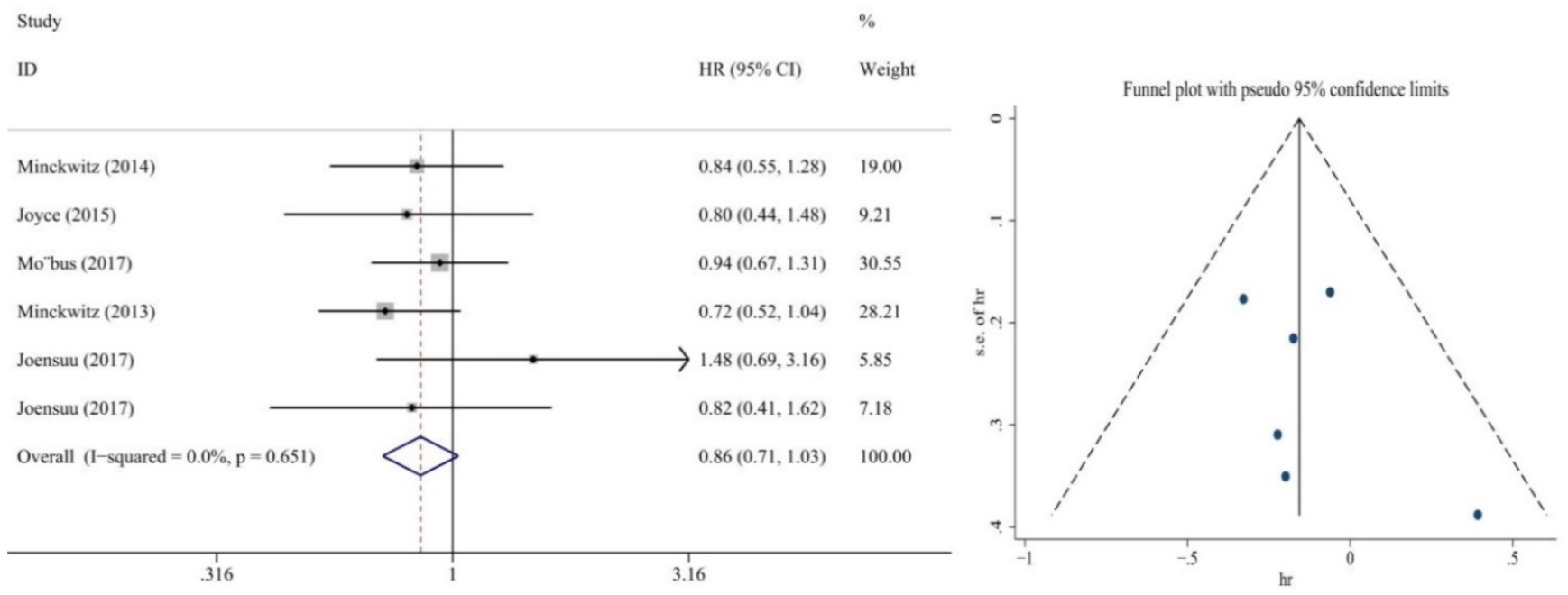

Figure 12. Forest plot and funnel chart of meta-analysis on the disease-free survival for Her2+ patients
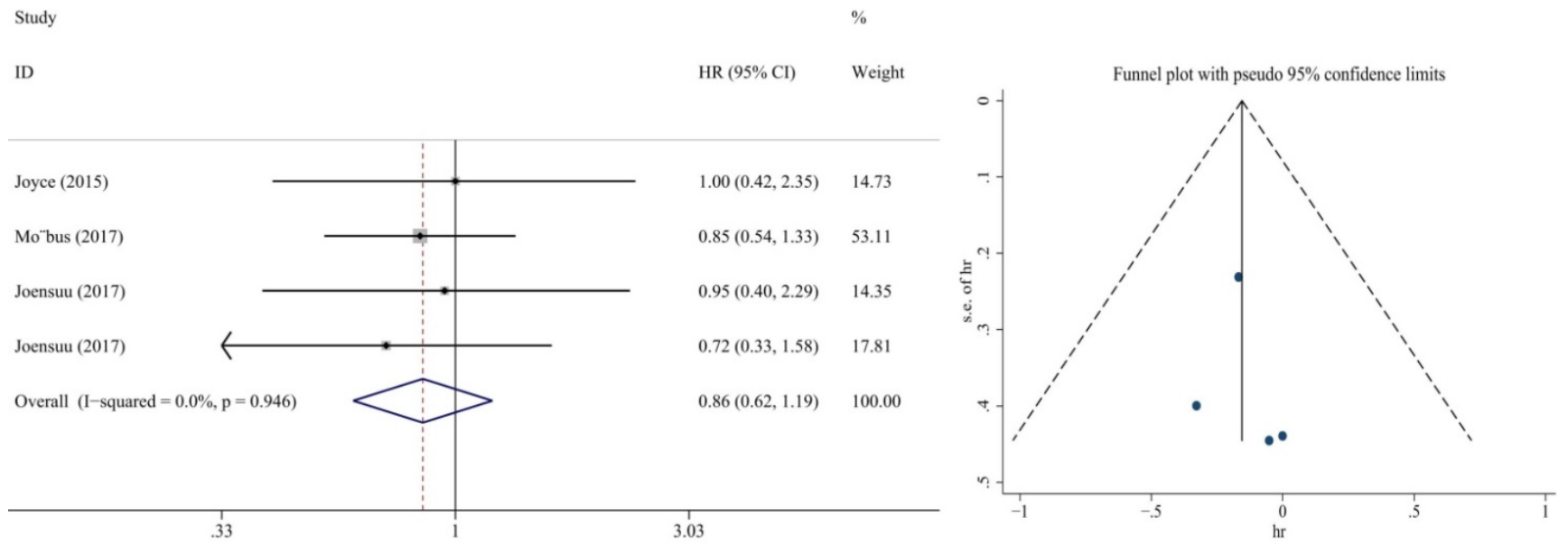

Figure 13. Forest plot and funnel chart of meta-analysis on the overall survival for Her2+ patients

Study

ID

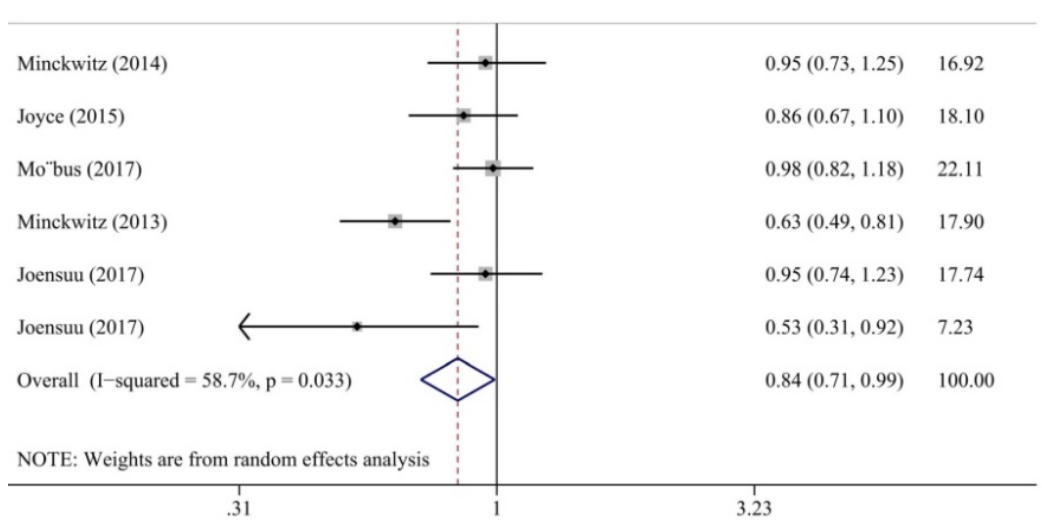

$\mathrm{HR}(95 \% \mathrm{CI}) \quad$ Weight

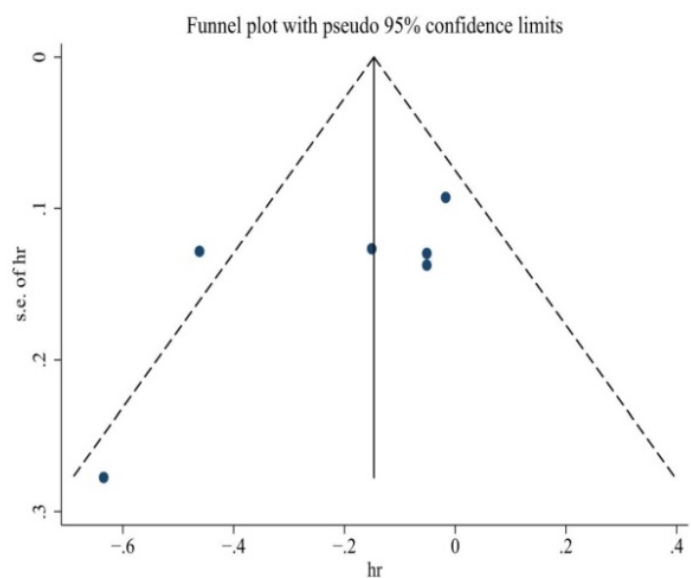

Figure 14. Forest plot and funnel chart of meta-analysis on the disease-free survival for Her2- patients 
Study

ID
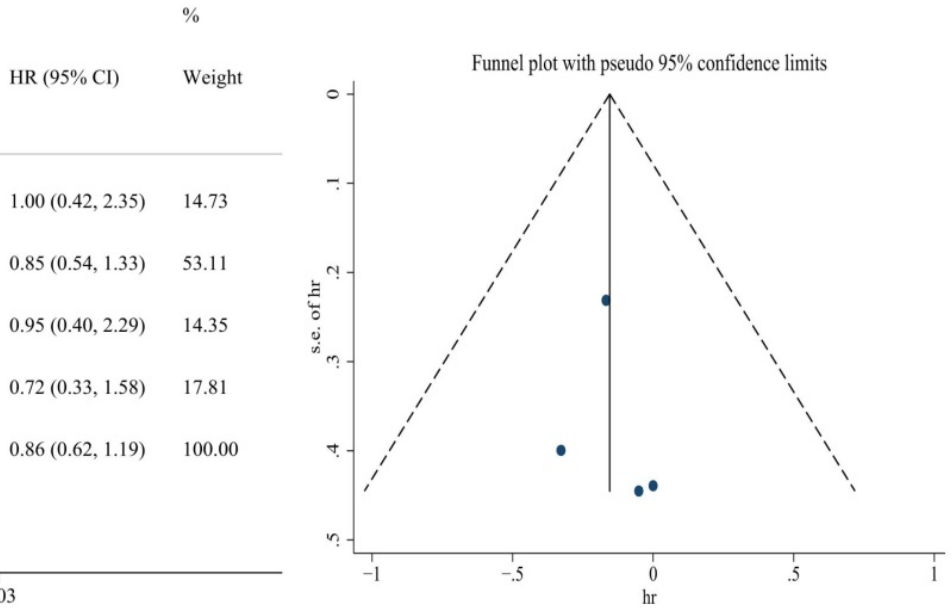

Figure 15. Forest plot and funnel chart of meta-analysis on the overall survival for Her2- patients
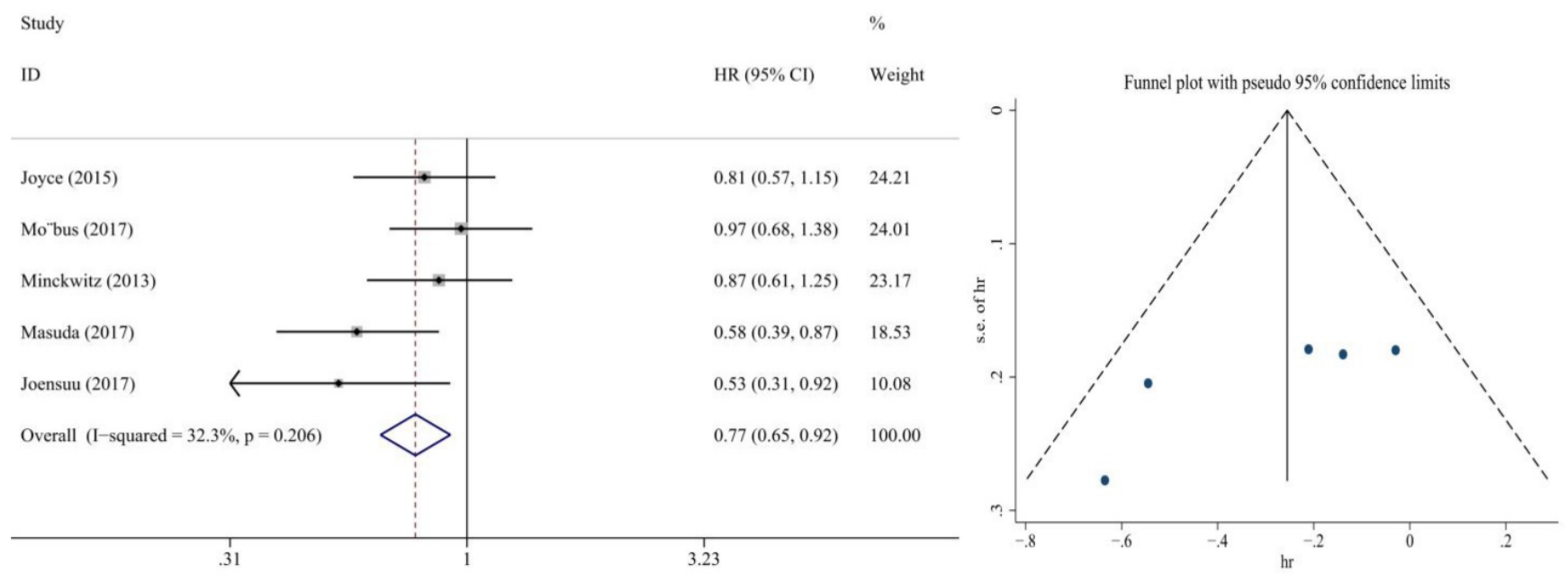

Figure 16. Forest plot and funnel chart of meta-analysis on the disease-free survival for TNBC patients
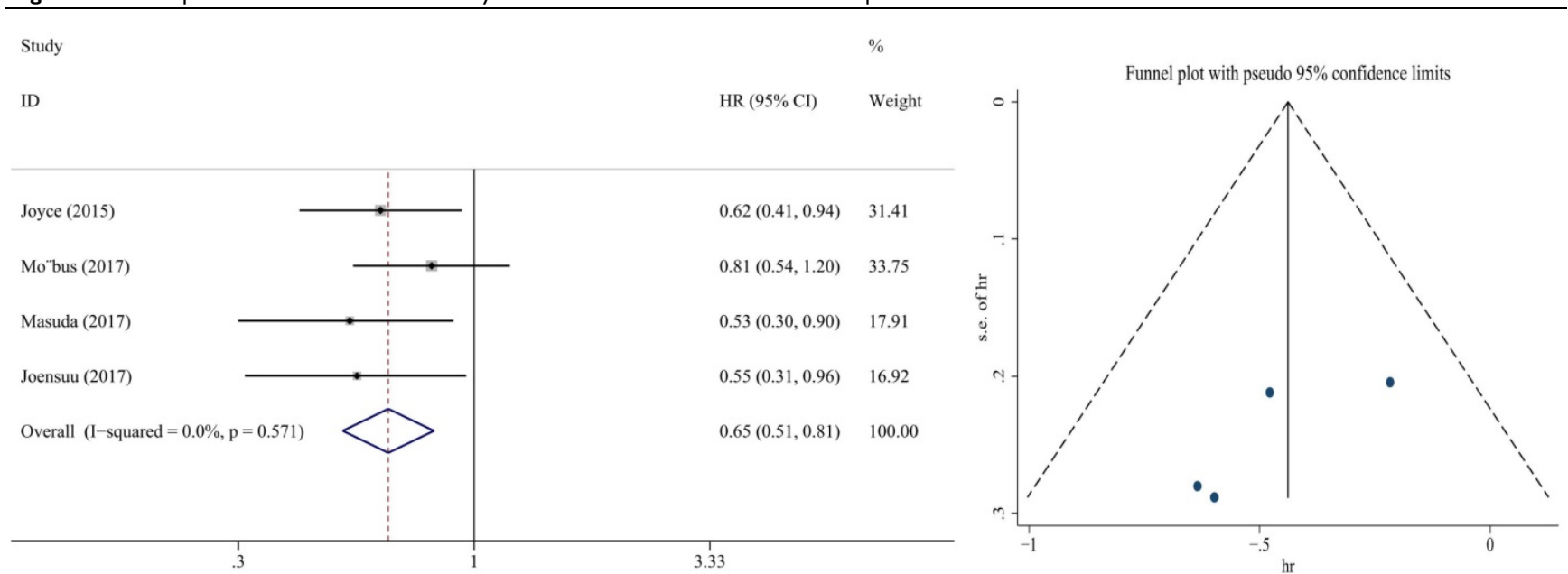

Figure 17. Forest plot and funnel chart of meta-analysis on the overall survival for TNBC patients

In non-hematologic toxicity, the adverse event diarrhea had significant difference in cap and non-cap group. It occured more frequently in capecitabine group than the non-capecitabine group ( $R R=2.1795 \%$ CI: 1.10-4.69; $\mathrm{P}=0.048$ ) (Figure 18). No publication bias was found.
In hematologic toxicity, we found that grade $3 / 4$ leucopenia was the most frequent serious adverse, it occurred more often in non-cap group than in cap group (RR=0.72 95\% CI: 0.59-0.86; $\mathrm{P}=0.000$ ) (Figure 19). No publication bias was found. 

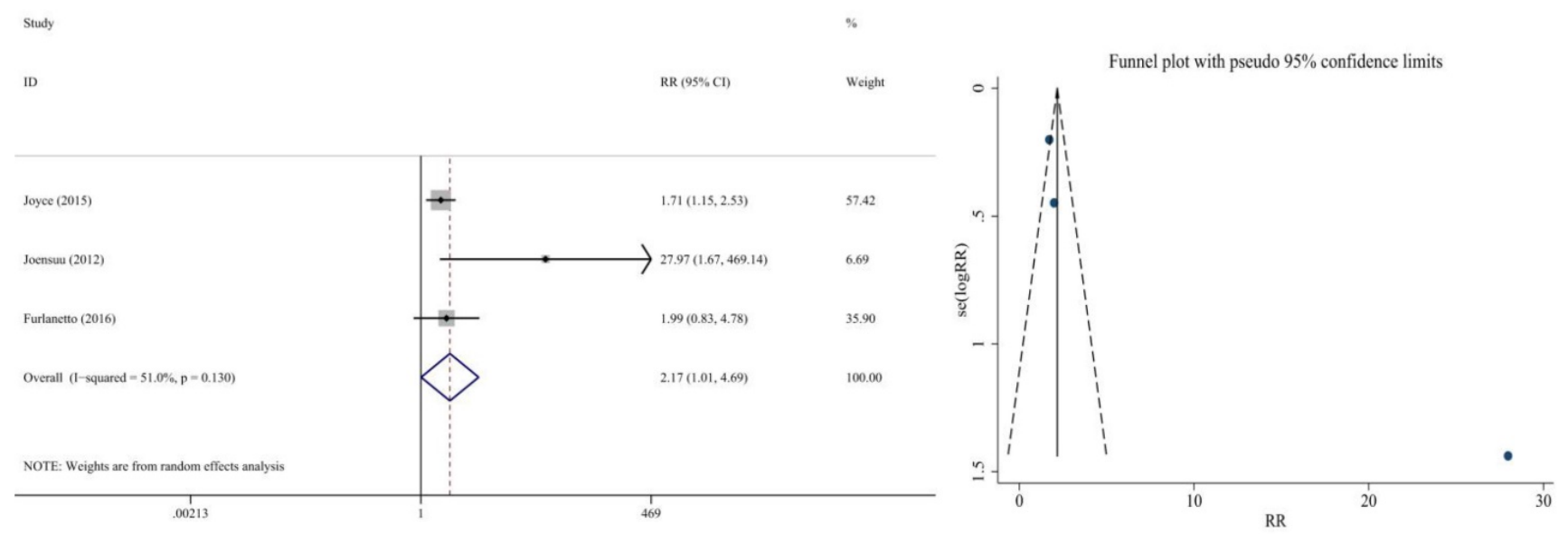

Figure 18. Forest plot and funnel chart of meta-analysis on the Incidence rate for diarrhea
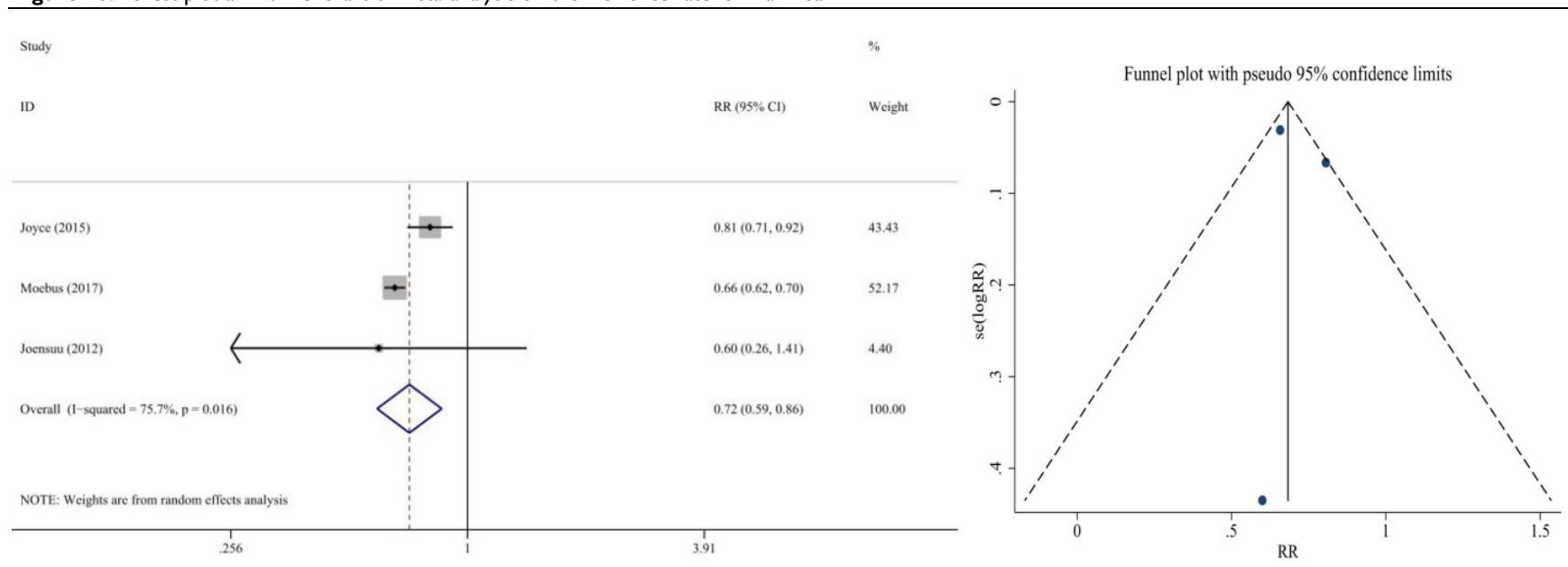

Figure 19. Forest plot and funnel chart of meta-analysis on the Incidence rate for leucopenia

The frequency of grade $3 / 4$ febrile neutropenia ( $R R=0.83$ 95\% CI: $0.35-1.96 ; \mathrm{P}=0.670)$, grade $3 / 4$ neutropenia ( $R R=0.9195 \% \mathrm{CI}$ : $0.55-1.50 ; \mathrm{P}=0.700)$, thrombocytopenia (RR=0.86 95\% CI: 0.02-30.17; $\mathrm{P}=$ $0.935)$, vomiting ( $R R=0.89$ 95\% CI: $0.61-1.30 ; \mathrm{P}=$ $0.561)$, anemia ( $R R=0.6595 \% \mathrm{CI}: 0.36-1.20 ; \mathrm{P}=0.168)$, hand-foot syndrome ( $R R=10.87$ 95\% CI: 0.03-4082.9; P $=0.430$ ) was similar in the cap and non-cap arms, respectively.

\section{Publication bias}

A funnel plot was applied to evaluate possible publication bias. Priority in positive results were reported concerning the addition of cap in the breast cancer therapies and more studies were needed to alleviate publication bias.

\section{Discussion}

The role of capecitabine in the treatment of advanced breast cancer has been recognized. However, it has not been confirmed that capecitabine combined with first-line chemotherapy can improve the survival of breast cancer patients. Our meta-analysis is the first to be combined with 10 phase 3 clinical controlled trials to assess the efficacy of addition of capecitabine with first-line chemotherapy in breast cancer. Also, we conducted subgroup analysis to compare the efficacy of capecitabine-based versus capecitabine-free combination neoadjuvant chemotherapy and adjuvant chemotherapy. Meanwhile, there are 6 articles included in each subanalysis of ER and Her2 status, which make the study of different subtype patients. However, there are a limited number of studies included in each sub-analysis. Consequently, our meta-analysis indicates that capecitabine-based combination first-line chemotherapy might provide survival benefits in $B C$ compared with capecitabine-free regimens, the endpoint DFS and OS have significant improvements.

In the past few decades, the importance of neoadjuvant therapy for breast cancer in the treatment of breast cancer has become increasingly clear. Clinical experts have conducted extensive research on the choice of neoadjuvant chemotherapy regimens, and whether capecitabine is a part of the standard neoadjuvant therapy is not yet clear [19]. Our 
meta-analysis delivered that even in the neoadjuvant and adjuvant subgroups, the survival benefit still has significant improvements in DFS and OS.

In a meta-analysis that evaluated the efficacy results of capecitabine with anthracycline- and taxane-based adjuvant therapy in early breast cancer, they pointed out that the addition of capecitabine at a HR of 0.83 (95\% CI: $0.71-0.98$ ) can significantly improve survival. Our meta-analysis find that the addition of capecitabine can improve disease-free survival $(\mathrm{HR}=0.84,95 \% \mathrm{CI}: 0.76-0.93 ; \mathrm{P} \approx 0.000)$ and overall survival $(\mathrm{HR}=0.84,95 \% \mathrm{CI}$ : $0.74-0.94 ; \mathrm{P}=$ 0.001), this is in consistent with the previous meta-analysis [20].

Also, the classification of tumors was analyzed for the three subgroups based on ER and HER2 status. Both in the ER-group, her2- group and TNBC group, the addition of capecitabine significantly improve DFS or OS. In conclusion, the addition of capecitabine in first-line chemotherapy prolonged disease-free survival and overall survival in patients with ER-negative or HER2-negative breast cancer. Worth emphasizing is the fact that the adjuvant capecitabine therapy showed effectiveness among patients in triple-negative subgroup which was complicated with treatment. However, the other two articles mentioned the significant benefit of capecitabine group in the triple negative subgroup by RFS, which cannot be combined with DFS $[11,14]$.

In terms of duration of treatment, the GeparQuattro study found that there was no difference in outcome for patients receiving 24 weeks or 36 weeks of chemotherapy $(\mathrm{P}=0.818$ for DFS and $\mathrm{P}=0.825)$ for OS [12].

Capecitabine is an oral, tumor-targeted drug, which was different from intravenous medication. In the safety of capecitabine combination therapy, only the incidence of some complications will increase such as diarrhea. The most frequent serious adverse event was febrile neutropenia, Grade 3 hand-foot syndrome, grade $3 / 4$ stomatitis and grade $3 / 4$ diarrhea, which were the same as non-combination first-line chemotherapy. However, the grade $3 / 4$ leucopenia occurred more often in non-cap group than in cap group ( $R R=0.7295 \% \mathrm{CI}: 0.59-0.86$; $\mathrm{P}=$ 0.000) [10-13], this may be related to the reduced dosage of docetaxel in capecitabine group [14].

Above all, our meta-analysis indicates that the addition of capecitabine in breast cancer first-line chemotherapy can significantly improve the survival of breast cancer patients regardless of neoadjuvant and adjuvant chemotherapy. In ER-, Her2- and TNBC patients, the addition of capecitabine will result in great benefits. We are looking forward to more clinical trials to explore the most appropriate dose of capecitabine for breast cancer.

\section{Competing Interests}

The authors have declared that no competing interest exists.

\section{References}

1. Siegel R L, Miller K D, Jemal A. Cancer statistics, 2018[J]. CA: A Cancer Journal for Clinicians. 2018; 60(5):277-300.

2. Miwa M, Ura M, Nishida M, et al. Design of a novel oral fluoropyrimidine carbamate, capecitabine, which generates 5-fluorouracil selectively in tumours by enzymes concentrated in human liver and cancer tissue[J]. European Journal of Cancer. 1998; 34(8):1274-81.

3. Blum J L, Jones S E, Buzdar A U, et al. Multicenter phase II study of capecitabine in paclitaxel-refractory metastatic breast cancer[J]. Journal of Clinical Oncology. 1999; 17(2):485-493.

4. Miles D, Von M G, Seidman A D. Combination versus sequential single-agent therapy in metastatic breast cancer[J]. Oncologist. 2002; 7 Suppl 6(Supplement 6):13-19.

5. O'Shaughnessy J, Miles D, Vukelja S, et al. Superior survival with capecitabine plus docetaxel combination therapy in anthracycline-pretreated patients with advanced breast cancer: phase III trial results[J]. Journal of Clinical Oncology. 2002; 20(12):2812-2823.

6. Blum J L, Dieras V, Lo Russo P M, et al. Multicenter, Phase II study of capecitabine in taxane-pretreated metastatic breast carcinoma patients[J]. Cancer. 2015; 92(7):1759-1768.

7. Jiang Y, Yin W, Zhou L, et al. First efficacy results of capecitabine with anthracycline- and taxane-based adjuvant therapy in high-risk early breast cancer: a meta-analysis[J]. Plos One. 2012; 7(3):e32474.

8. Li Q, Jiang Y, Wei W, et al. Clinical Efficacy of Including Capecitabine in Neoadjuvant Chemotherapy for Breast Cancer: A Systematic Review and Meta-Analysis of Randomized Controlled Trials[J]. Plos One. 2013; 8(1):e53403.

9. Von M G, Blohmer J U, Costa S D, et al. Response-guided neoadjuvant chemotherapy for breast cancer[J]. Journal of Clinical Oncology. 2013; 31(29):3623.

10. O'Shaughnessy J A, Koeppen $\mathrm{H}$, Xiao $\mathrm{Y}$, et al. Patients with Slowly Proliferative Early Breast Cancer have Low 5-Year Recurrence Rates in a Phase III Adjuvant Trial of Capecitabine[J]. Clinical Cancer Research An Official Journal of the American Association for Cancer Research. 2015; 21(19):4305.

11. Masuda N, Lee S J, Ohtani S, et al. Adjuvant Capecitabine for Breast Cancer after Preoperative Chemotherapy.[J]. N Engl J Med. 2017; 376(22):2147-2159.

12. Von M G, Rezai M, Fasching P A, et al. Survival after adding capecitabine and trastuzumab to neoadjuvant anthracycline-taxane-based chemotherapy for primary breast cancer (GBG 40--GeparQuattro)[J]. Annals of Oncology Official Journal of the European Society for Medical Oncology. 2014; 25(1):81-9.

13. Von M G, Möbus V, Schneeweiss A, et al. German adjuvant intergroup node-positive study: a phase III trial to compare oral ibandronate versus observation in patients with high-risk early breast cancer[J]. Journal of Clinical Oncology. 2013; 31(28):3531-3539.

14. Joensuu H, Kellokumpu-Lehtinen P L, Huovinen R, et al. Adjuvant capecitabine, docetaxel, cyclophosphamide, and epirubicin for early breast cancer: final analysis of the randomized FinXX trial[J]. Journal of Clinical Oncology. 2012; 30(1):11-18.

15. Joensuu $\mathrm{H}$, Kellokumpu-Lehtinen $\mathrm{P}$ L, Huovinen $\mathrm{R}$, et al. Adjuvant Capecitabine in Combination With Docetaxel, Epirubicin, and Cyclophosphamide for Early Breast Cancer: The Randomized Clinical FinXX Trial[J]. Jama Oncology. 2017; 3(6).

16. Furlanetto J, Eiermann W, Marmé F, et al. Higher Rate of Severe Toxicities in Obese Patients Receiving dose-dense (dd) Chemotherapy according to Unadjusted Body Surface Area- Results of the Prospectively Randomized GAIN study[J]. Annals of Oncology Official Journal of the European Society for Medical Oncology. 2016; 27(11):2053.

17. Chow L W, Nakamura S. Randomized trial of preoperative docetaxel with or without capecitabine after 4 cycles of 5-fluorouracil-epirubicincyclophosphamide (FEC) in early-stage breast cancer: exploratory analyses identify $\mathrm{Ki} 67$ as a predictive biomarker for response to neoadjuvant chemotherapy. [J]. Breast Cancer Research and Treatment. 2013; 142(1):69-80.

18. Hatschek T, Carlsson L, Einbeigi Z, et al. Individually tailored treatment with epirubicin and paclitaxel with or without capecitabine as first-line chemotherapy in metastatic breast cancer: a randomized multicenter trial.[J]. Breast Cancer Research \& Treatment. 2012; 131(3):939-947.

19. Rubovszky G, Horváth Z. Recent Advances in the Neoadjuvant Treatment of Breast Cancer.[J]. Journal of Breast Cancer. 2017; 20(2):119-131.

20. Jiang Y, Yin W, Zhou L et al.. First efficacy results of capecitabine with anthracycline- and taxane-based adjuvant therapy in high-risk early breast cancer: a meta-analysis. PLoS One. 2012; 7(3): e32474 
21. Von M G, Rezai M, Fasching P A, et al. Survival after adding capecitabine and trastuzumab to neoadjuvant anthracycline-taxane-based chemotherapy for primary breast cancer (GBG 40--GeparQuattro)[J]. Annals of Oncology

Official Journal of the European Society for Medical Oncology. 2014; 25(1):81-9. 